\title{
Chemical Sensors for Indoor Applications
}

\author{
Andreas Schütze \\ Lab for Measurement Technology, Dept. of Mechatronics, Saarland University, 66123 Saarbrücken, \\ Germany \\ schuetze@LMT.uni-saarland.de
}

\begin{abstract}
:
People spend an increasing amount of their time indoors. Thus, environmental conditions and air quality but also safety aspects are of specific concern in indoor settings. This contribution gives an overview of chemical sensor technologies used for indoor applications, focusing on explosive gas leak and fire detection as well as indoor air quality as the most relevant applications. These applications are traditionally addressed with a variety of technologies, such as metal-oxide semiconductor gas sensors for gas leak detection or infrared sensors for $\mathrm{CO}_{2}$ monitoring. Increased requirements led to multisensor developments especially for security applications such as fire detection which demand a high reliability of the sensor system. In recent years, modern signal processing techniques have increasingly been used to overcome typical problems of chemical sensors, especially limited selectivity as well as drift and aging. Some examples for state-of-the-art chemical sensor systems for indoor applications are given showing the growing potential in this area. Novel developments such as gas sensitive field effect transistors (GasFETs), which are currently commercially introduced, and sensor self-monitoring strategies for increased reliability are discussed as well as trends for the future.
\end{abstract}

Key words: gas leak detection, indoor air quality, fire detection, semiconductor gas sensor, GasFET, virtual multisensor

\section{Introduction}

Indoor applications are an area with increasing interest in the field of chemical sensors. One driver is the increased demand for safety, not only in public buildings but also in private homes. In this context, detection of explosive gas leaks and early fire detection are still issues requiring optimal solutions, even though much progress has been made in the past. A second, more recent area of interest is the control of HVAC (heating, ventilation, air conditioning) equipment as heating and cooling accounts for a large percentage of the primary energy used today. Demands for reduced energy consumption can only be met when air circulation is reduced, thus buildings tend to become more and more air tight. However, this adversely affects the health of the inhabitants due to increased levels of carbon monoxide $\left(\mathrm{CO}_{2}\right)$ correlating with human presence but also volatile organic compounds (VOC) which result from a wide spectrum of sources, such as building materials, furniture, cleaning agents and also human activity (i.e. sweat). Increasing concern especially regarding the sick building syndrome has led to demand for chemical sensor systems which can detect the most relevant components for determining indoor air quality (IAQ) in order to minimize energy consumption without causing adverse effects for humans in the indoor environment. Note that this should also take the specific scenario, i.e. the type of indoor use, into account: work environments will require different solutions compared to living rooms and bedrooms, kitchens and bathrooms, as the time spent in each scenario and the atmospheric composition vary widely.

Many sensor principles have been tested and are used in indoor applications, notably infrared (IR) detection, especially for $\mathrm{CO}_{2}$ monitoring, as well as electrochemical cells (EC), e.g. for carbon monoxide (CO) detection for fire alarms and also to prevent poisoning by defective heating units. However, the most successful sensor principle for indoor use remains the metal oxide semiconductor (MOS) gas sensor. Indeed, the original Figaro sensors were specifically developed for addressing an indoor application, namely explosive gas leak detection, which is required by law in Japan. The unique selling point of these sensors, apart from their low cost combined with high sensitivity, is their unrivaled robustness: MOS sensors have proven reliable over more than ten years of continuous operation. This is a very important point for indoor applications, were sensor replacement every few months is not acceptable. 


\section{Gas measurement systems}

For selection and optimization of chemical sensors, the three "S" are the primary challenges:

Sensitivity describes the requirement that the sensor must detect the relevant gas or gas mixture at the concentration appropriate for the desired application. This varies widely, even in indoor applications, from several $1000 \mathrm{ppm}$, e.g. for $\mathrm{CO}_{2}$ detection (400 to $5000 \mathrm{ppm}$ ) or explosive gas leak detection $\left(\mathrm{CH}_{4}\right.$ warning level typically 4000 ppm) down to ppb levels, e.g. for hazardous gases like ozone (limits range from 50 to $300 \mathrm{ppb}$ ) or formaldehyde (the limit has recently been lowered from 500 to $300 \mathrm{ppb}$ ), but also trace gases for early fire detection.

Selectivity is currently the main R\&D challenge for gas measurement systems as most sensors - and especially MOS sensors - show a wide response spectrum with many cross sensitivities. Ideally, a sensor should only react to a specific target gas or situation. Selectivity can be optimized by selecting material, sensor layer preparation and proper operating conditions, e.g. temperature. Note however, that a sensor with wide response spectrum also offers the opportunity for addressing multiple applications with a single sensor.

Stability is often overlooked as a critical challenge for chemical sensor systems, at least in research projects which tend to concentrate on increasing sensitivity and/or selectivity. However, to be able to address real world problems, stability of the sensor is a must. Indeed, for applications in safety and security, stability is today seen as the issue preventing the widespread application of many sensor technologies - especially MOS sensors. Note that robustness on the one hand and stability on the other are two quite distinct issues: while MOS sensors are known to operate over many years, reliable identification and/or quantification of gases is often difficult due to drift, aging or poisoning of the gas sensitive layer.

While the chemical sensor is of course a highly relevant part of any gas measurement system, it has to be noted that the sensor element alone will in general not be able to address all application specific challenges. A generic scheme for gas measurement systems is shown in Fig. 1. The depicted modular approach emphasizes the importance of electronics for operation of the sensor and data acquisition as well as subsequent signal processing and evaluation.

These systems often also contain additional sensors, e.g. for relative humidity (r.h.) or temperature $(T)$, which can be used in multiple ways. Internally, the data from these sensors can be used to correct the information obtained from the chemical sensors, i.e. to suppress cross interference effects or linearize the response for quantitative measurements. Externally, these sensors can provide additional data for the overall application. Temperature and humidity are obviously important parameters for indoor climate in HVAC systems, but temperature sensors are also used for fire detection.

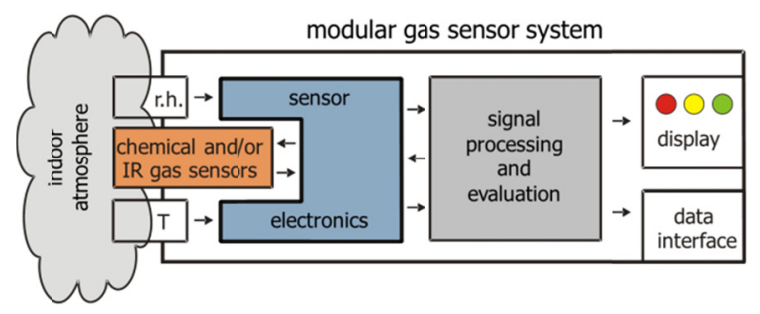

Fig.1.Schematic of a modular sensor system for indoor applications.

Signal processing has gained huge interest in the field of chemical sensors in recent years with the advance of multisensor systems, which were developed primarily to address the limited selectivity of single sensors. In many modern applications multiple chemical sensors are used combined with pattern recognition tools in order to achieve amazing selectivity, which can e.g. allow differentiation of different types of fire and non-fire situations to prevent false alarms in fire detection applications [1,2] .

In addition to multisensor systems, dynamic operation of a single (or only a few) chemical sensors has also proven very successful in addressing the three "S". Temperature cycled operation (TCO) [3] and electrical impedance spectroscopy (EIS) [4] have both proven highly successful especially for MOS sensors. Note that this dynamic operation of a single sensor, also called a virtual multisensor [5], not only increases the selectivity but can also improve the stability. While for a multisensor array drift of a single sensor can already prevent correct evaluation, virtual multisensors haven proven stable over periods of several months in the lab and various field tests $[6,7]$.

\section{Calibration and field testing}

Another aspect that is easily overlooked for development of gas measurement systems is the issue of testing and calibration. For fire detection the current norms describe not only the requirements of the detectors themselves but also the scenarios for their testing [8]. However, these currently focus on smoke detectors and are therefore somewhat ill-suited for fire detectors based on chemical sensors. For development of signal processing techniques which are often based on multivariate, i.e. statistical, data analysis [9] the standard tests are very time consuming and expensive. 
Fig. 2 shows a miniaturized test set-up for efficient development of fire detectors based on chemical sensors [10]. With this system, a gas measurement system for fire detection was developed based on a single microstructured MOS sensor combined with TCO and intelligent signal processing. The average heater power consumption of the system was only $1 \mathrm{~mW}$ allowing operation of the system in a data bus powered network environment $[10,11]$.

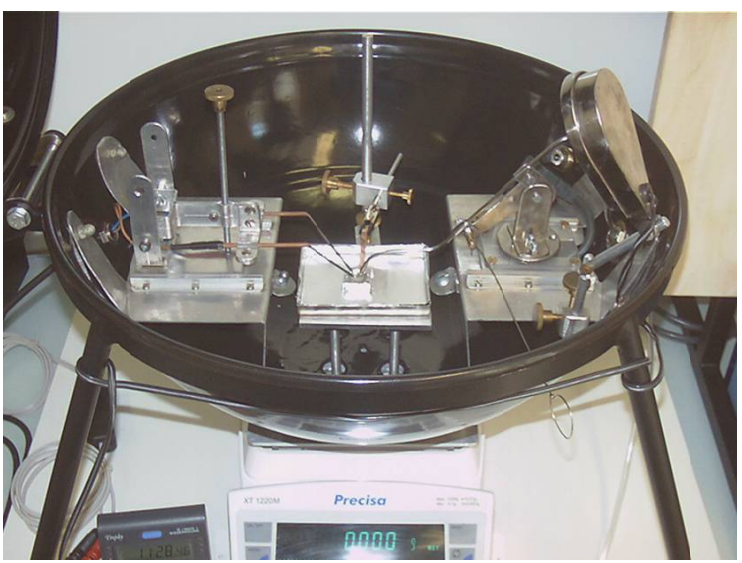

Fig. 2. Miniaturized test chamber for simulating fire situations. The test system is based on EN54 [8], test fires are scaled to obtain similar gas concentrations. A single test can be performed in 30 to $90 \mathrm{~min}$. [10]

This gas measurement system was subsequently used in field tests in various challenging operating environments such as chicken pens, chemical labs and cafeteria kitchens [11]. Tests over several months, cf. Fig. 3, have proven the concept but did also show the requirement for recalibration or at least sensor self-monitoring to identify drift or poisoning effects which would prevent correct classification of the alarm.

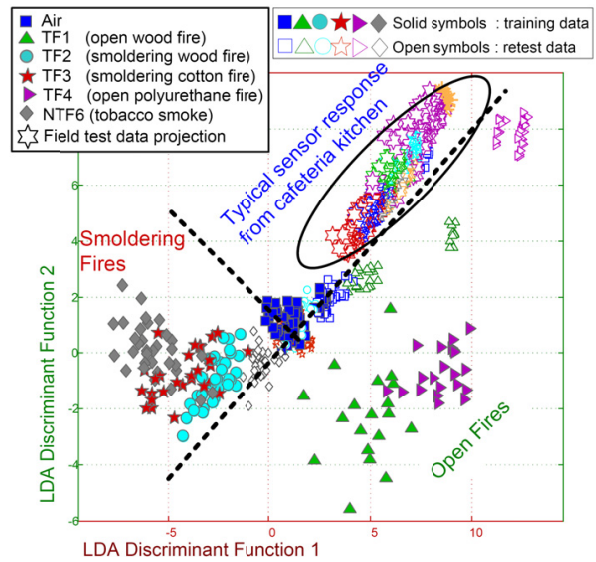

Fig.3.LDA result of the long term field test in a cafeteria kitchen. Sensor response caused by normal kitchen activities can be discriminated from trained alarm situations. [10]

\section{Novel developments}

The increasing demand for more versatile and reliable sensor systems has led to research in many fields. In recent years, nanotechnology has become a hot topic for further improving and custom tailoring gas sensitive layers [12].

For improving selectivity, the virtual multisensor approach using TCO was also extended to other sensor principles such as pellistors [13] or GasFETs [14]. Recently, a novel approach to sensor self-monitoring was presented by combining TCO and EIS in a single system $[15,16]$. Fig. 4 shows the fundamental concept which is currently being studied in lab tests for various applications in safety and security.

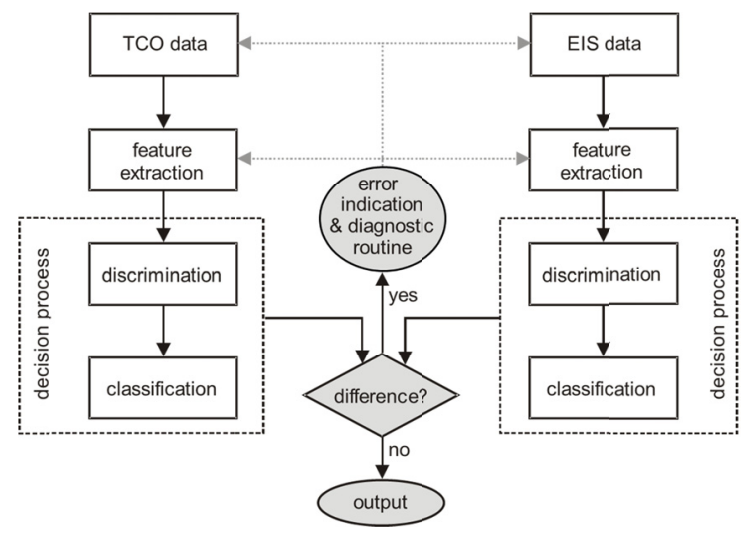

Fig.4.Fundamental approach to sensor self-monitoring based on parallel dynamic operation of a single MOS gas sensor with TCO and EIS plus independent evaluation. Different classificiation results contradict the calibration indicating a defective sensor. [16]

Recently, GasFETs, which have been under development for many years since the concept was first described by Lundström [17], were introduced on a large sicale for commercial applications, cf. Fig. 5 and 6.This extends the available gas sensor spectrum for indoor applications, especially because gas sensitive layer and transducer are decoupled in this approach.

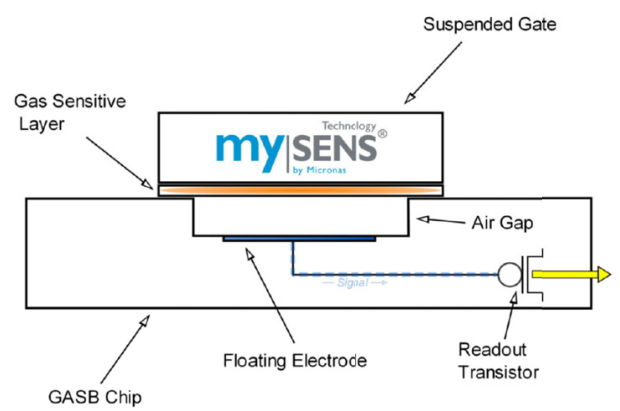

Fig. 5. Function principle of a gas sensitive CCFET (Capacitive Coupled Field Effect Transistor); gas molecules adsorb on the gas sensitive layer, the resulting shift of the surface potential is read out via a floating electrode (source: Micronas $\mathrm{GmbH}$ ). 


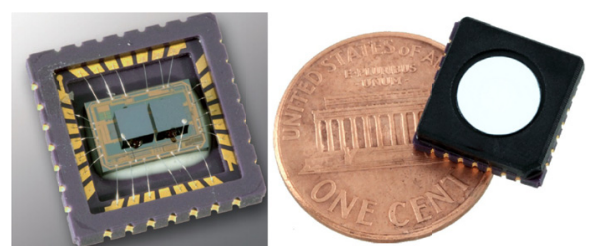

Fig. 6. Integrated sensor chip with two CCFETs; the suspended gates with gas specific material are bonded onto the chip separately (source: Micronas).

The novel technology combines microelectronic technology, suitable for large volume, low cost production as well as integration of signal processing, with a broad gas detection spectrum due to the flexible integration of gas sensitive layers deposited on the suspended gates. For indoor applications, this technology is suitable for fire detection as well as air quality monitoring. It is very interesting to note that even for $\mathrm{CO}_{2}$ promising gas sensitive layers have been developed, which would allow IAQ applications without the costly IR measurement cell currently often used [18]. However, stability in long term operation still needs to be ascertained.

\section{Conclusion and outlook}

Chemical sensors are expected to become ubiquitous in modern indoor applications increasing safety (e.g. for fire and gas leak detection) and reducing energy consumption by optimized HVAC operation without lowering comfort or health standards. Intelligent gas measurement systems combining state-of-the-art sensors with dynamic operation and signal processing will moreover offer the possibility to address multiple applications simultaneously, e.g. IAQ and fire detection, thus reducing cost, especially in modern networked building environments.

\section{Acknowledgements}

Many co-workers at LMT have contributed to the results presented here, their contributions over the last 12 years are gratefully acknowledged. Fruitful cooperation with many researchers from academia as well as industrial partners from sensor manufacturers, systems integrators and end-users is also gratefully acknowledged. The lively discussions on many issues have contributed to our work in many aspects. Special thanks also to I. Freund, Micronas, for supplying material and figures describing the novel GasFET technology.

\section{References}

[1] D. Gutmacher et al., Gas sensor technologies for fire detection, Sens. Actuators B: Chem. (2011), doi: 10.1016/j.snb.2011.11.053

[2] J. Kelleter et al., Gas-Sensor-Melder für Schwelbrände, s+s report, Nr. 3 (1995).
[3] A.P. Lee, B.J. Reedy, Temperature modulation in semiconductor gas sensing, Sens. Actuators B: Chem., 60(1999), 35-42.

[4] U. Weimar, W. Göpel, A.c. measurements on tin oxide sensors to improve selectivities and sensitivities, Sens. Actuators B: Chem., 2627(1995), 13-18.

[5] A. Schütze, A. Gramm, T. Rühl, Identification of Organic Solvents by a Virtual Multisensor System with Hierarchical Classific:ation, IEEE Sensors J, vol. 4, no. 6 (2004), 857-863.

[6] A. Gramm, A. Schütze, High performance solvent vapor identification with a two sensor array using temperature cycling and pattern classification, Sens. Actuators B: Chem., 95 (2003), 58-65.

[7] S. Horras et al., Correlation of an E-Nose system for odor assessment of shoe/socks systems with a human sensory panell, Proc. ISOEN Conf. 2009, Brescia, Italy, April 15 to 17

[8] European Standard EN 54-7:2006-09 (point type smoke detectors) and EN54-9:1984 (fire sensitivity tests); Beuth-Verlag

[9] R. Gutierrez-Osuna, Pattern Analysis for Machine Olfaction: a Review, IEEE Sensors J, vol. 2, no. 3 (2002), 189-202.

[10] Z. Ankara: UntersuchungzumEinsatz von mikrostrukturiertenHalbleitergasisensoren in der heutigenGebäudebrandmeldetechnik, Dissertation, Saarland University (20108), Shaker, Aachen, ISBN 978-3-8322-7310-1

[11] Z. Ankara, A. Schütze, Low Power Virtual Sensor System based on a Micromachined Gas Sensor for Security Applications and Warning Systems, Proc. EUROSENSORS XXII Conf. (2008), Dresden, Germany, September 7-10.

[12] Cf. special session on Cost Action EuNetAir "Sensor materials and Techniques", Proc. IMCS (2012), Nuremberg, Germany,May 20-23.

[13] M. Engel, UntersuchungzurKalibrierbarkeit von GassensorenimtemperaturzyklischenBetrieb, Dissertation, Saarland University (2008), Shaker, Aachen, ISBN 978-3-8322-7446-7

[14] C. Bur et al., New method for selectivity enhancement of $\mathrm{SiC}$ field effect gas sensors for quantification of NOx, Microsystem Technologies(2012) in print, doi: 10.1007/s00542-012-1434-z

[15] P. Reimann, A. Dausend, A. Schütze, A selfmonitoring and self-diagnosis strategy for semiconductor gas sensor systems, Proc. IEEE Sensors Conf. (2008), Lecce, Italy, Oct. 27 - 29.

[16] P. Reimann et al., Improving MOS Virtual Multisensor Systems by Combining Temperature Cycled Operation with Impedance Spectroscopy, Proc. ISOEN 2011, AIP Conf. Proc. 1362 (2011), 249-250; doi: 10.1063/1.3626378

[17] I. Lundström et al., A hydrogen sensitive MOS field effect transistor, Appl. Phys. Let., 26 (1975), 55-57.

[18] S. Stegmeier et al., Stepwise improvement of (hetero-)polysiloxane sensing layers for $\mathrm{CO}_{2}$ detectionoperated at room temperature by modification of the polymeric network, Sens. 
Actuators B: Chem. 148(2010), 450-458, doi: 10.1016/j.snb.2010.05.016 